\title{
Spectral phase conjugation with cross-phase modulation compensation
}

\author{
Mankei Tsang and Demetri Psaltis \\ Electrical Engineering Department, California Institute of Technology, Pasadena, California \\ 91125 \\ mankei@sunoptics.caltech.edu
}

\begin{abstract}
Spectral phase conjugation with short pump pulses in a third-order nonlinear material is analyzed in depth. It is shown that if signal amplification is considered, the conversion efficiency can be significantly higher than previously considered, while the spectral phase conjugation operation remains accurate. A novel method of compensating for cross-phase modulation, the main parasitic effect, is also proposed. The validity of our theory and the performance of the spectral phase conjugation scheme are studied numerically.
\end{abstract}

(C) 2004 Optical Society of America

OCIS codes: (190.5040) Phase conjugation; (190.4380) Nonlinear optics, four-wave mixing.

\section{References and links}

1. D. A. B. Miller, "Time reversal of optical pulses by four-wave mixing," Opt. Lett. 5, 300 (1980).

2. C. Joubert, M. L. Roblin, and R. Grousson, "Temporal reversal of picosecond optical pulses by holographic phase conjugation,” Appl. Opt. 28, 4604 (1989).

3. M. Tsang and D. Psaltis, "Dispersion and nonlinearity compensation by spectral phase conjugation," Opt. Lett. 28, 1558 (2003).

4. N. W. Carlson, L. J. Rothberg, A. G. Yodh, W. R. Babbitt, and T. W. Mossberg, "Storage and time reversal of light pulses using photon echoes," Opt. Lett. 8, 483 (1983).

5. V. L. da Silva, Y. Silberberg, J. P. Heritage, E. W. Chase, M. A. Saifi, and M. J. Andrejco, "Femtosecond accumulated photon echo in Er-doped fibers," Opt. Lett. 16, 1340 (1991).

6. A. Rebane, J. Aaviksoo, and J. Kuhl, "Storage and time reversal of femtosecond light signals via persistent spectral hole burning holography," Appl. Phys. Lett. 54, 93 (1989).

7. S. Fraigne, J. P. Galaup, J. L. Le Gouet, B. Bousquet, L. Canioni, M. Joffre, and J. P. Likforman, "Amplitude and phase measurements of femtosecond pulses shaped by use of spectral hole burning in free-base naphthalocyaninedoped films," J. Opt. Soc. Am. B 20, 1555 (2003).

8. A. M. Weiner, D. E. Leaird, D. H. Reitze, and Eung Gi Paek, "Femtosecond spectral holography," IEEE J. Quantum Electron. 28, 2251 (1992).

9. D. Marom, D. Panasenko, R. Rokitski, P. Sun, and Y. Fainman, "Time reversal of ultrafast waveforms by wave mixing of spectrally decomposed waves," Opt. Lett. 25, 132 (2000).

10. S. Wen, S. Chi, abd T. Chang, "Effect of cross-phase modulation on optical phase conjugation in dispersionshifted fiber," Opt. Lett. 19, 939 (1994).

11. R. Quintero-Torres and M. Thakur, "Measurement of the nonlinear refractive index of polydiacetylene using Michelson interferometry and z-scan,” J. Appl. Phys. 85, 401 (1999).

12. A. M. Weiner, "Femtosecond pulse shaping using spatial light modulators," Rev. Sci. Instrum. 71, 1929 (2000).

\section{Introduction}

Spectral phase conjugation (SPC) [1] is the phase conjugation of individual spectral components of an optical waveform, which is equivalent to phase conjugation and time reversal of the pulse envelope. Joubert et al. prove that midway SPC can compensate for all chromatic 
dispersion [2]. In a recent paper we prove that midway SPC can simultaneously compensate for self-phase modulation (SPM), self-steepening and dispersion [3]. The physical implementation of SPC is first suggested by Miller using short-pump four-wave mixing (FWM) [1], and later demonstrated using photon echo $[4,5]$, spectral hole burning $[6,7]$, temporal holography [2], spectral holography [8] and spectral three-wave mixing (TWM) [9]. The FWM scheme is especially appealing to real-world applications such as communications and ultrashort pulse delivery due to its simple setup. However, low conversion efficiency and parasitic Kerr effects make a practical implementation difficult.

In this paper we derive an accurate expression for the output idler when the conversion efficiency, defined as the output idler energy divided by the input signal energy, is high. We prove that if signal amplification is considered, the SPC process remains intact and the conversion efficiency can grow exponentially with respect to the cross fluence of the two pump pulses, compared with a quadratic growth predicted in Ref. [1].

As the theoretical conversion efficiency approaches $100 \%$, which is required for the purpose of nonlinearity compensation, parasitic effects begin to hamper the efficiency and accuracy of SPC. The main parasitic effect is cross-phase modulation (XPM) due to the strong pump, a problem that similarly plagues conventional temporal phase conjugation schemes [10]. We suggest a novel method to compensate for XPM by adjusting the phases of the pump pulses appropriately. We show that in theory, this method can fully compensate for the XPM effect.

Finally, numerical analysis is performed to confirm our predictions about the conversion efficiency and XPM compensation. Pump depletion is also addressed by full three-dimensional simulations.

\section{Spectral phase conjugation by four-wave mixing}

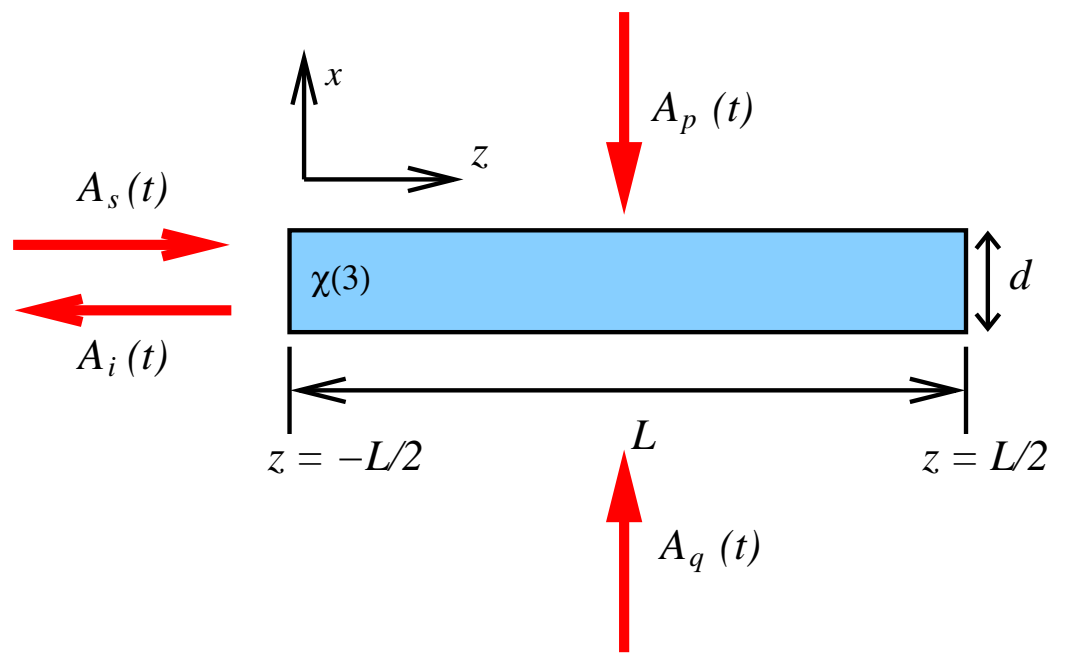

Fig. 1. Setup of SPC by four-wave mixing. $A_{s}(t)$ is the signal pulse, $A_{p}(t)$ and $A_{q}(t)$ are the pump pulses, and $A_{i}(t)$ is the backward-propagating idler pulse (After Ref. [1]).

The configuration of spectral phase conjugation by four-wave mixing introduced in Ref. [1] is drawn in Fig. 1. $A_{p}$ and $A_{q}$ are the envelopes of the pump pulses propagating downward and upward respectively, $A_{s}$ is the forward-propagating signal envelope, and $A_{i}$ is the backwardpropagating idler envelope. The coupled-mode equations that govern $A_{p}, A_{q}, A_{s}$ and $A_{i}$ can be 
derived from the wave equation and are given by

$$
\begin{aligned}
-\frac{\partial A_{p}}{\partial x}+\frac{1}{v_{x}} \frac{\partial A_{p}}{\partial t} & =j \gamma\left[2 A_{s} A_{i} A_{q}^{*}+\left(\left|A_{p}\right|^{2}+2\left|A_{q}\right|^{2}+2\left|A_{s}\right|^{2}+2\left|A_{i}\right|^{2}\right) A_{p}\right], \\
\frac{\partial A_{q}}{\partial x}+\frac{1}{v_{x}} \frac{\partial A_{q}}{\partial t} & =j \gamma\left[2 A_{s} A_{i} A_{p}^{*}+\left(2\left|A_{p}\right|^{2}+\left|A_{q}\right|^{2}+2\left|A_{s}\right|^{2}+2\left|A_{i}\right|^{2}\right) A_{q}\right], \\
\frac{\partial A_{s}}{\partial z}+\frac{1}{v} \frac{\partial A_{s}}{\partial t} & =j \gamma\left[2 A_{p} A_{q} A_{i}^{*}+\left(2\left|A_{p}\right|^{2}+2\left|A_{q}\right|^{2}+\left|A_{s}\right|^{2}+2\left|A_{i}\right|^{2}\right) A_{s}\right], \\
-\frac{\partial A_{i}}{\partial z}+\frac{1}{v} \frac{\partial A_{i}}{\partial t} & =j \gamma\left[2 A_{p} A_{q} A_{s}^{*}+\left(2\left|A_{p}\right|^{2}+2\left|A_{q}\right|^{2}+2\left|A_{s}\right|^{2}+\left|A_{i}\right|^{2}\right) A_{i}\right], \\
\gamma & =\frac{3 \omega_{0} \chi^{(3)}}{8 c n_{0}},
\end{aligned}
$$

where $v_{x}$ and $v$ are group velocities in the $x$ direction and the $z$ direction respectively and $n_{0}$ is the refractive index. Diffraction and group-velocity dispersion are neglected. The spatial dependence of $A_{p}$ on $z$ can also be suppressed if the illumination is uniform in $z$ and undepleted. If we further assume that the thickness of the medium $d$ is much smaller than the pump pulse width, then the dependence on the $x$ dimension can also be neglected.

The zeroth-order solution is the linear propagation of the incoming waves. Let the zerothorder solution be

$$
\begin{aligned}
& A_{p}^{(0)}(x, t)=A_{p}(t), \\
& A_{q}^{(0)}(x, t)=A_{q}(t), \\
& A_{s}^{(0)}(z, t)=F\left(t-\frac{z}{v}\right), \\
& A_{i}^{(0)}(z, t)=0 .
\end{aligned}
$$

The first-order solution can then be obtained by substituting the zeroth-order solution into the right-hand side of Eqs. (3) and (4). Each of Eqs. (1)-(4) has a single wave mixing term (first term on the right-hand side) and four phase modulation terms, which generally distort the pulses. With the subsitutions only Eq. (4) has a non-zero wave mixing term, and the output idler $A_{i}\left(-\frac{L}{2}, t\right)$ in the first order is shown to be the SPC of the input signal [1],

$$
A_{i}^{(1)}\left(-\frac{L}{2}, t\right)=j F^{*}\left(-t+\frac{L}{2 v}\right) \int_{-\infty}^{\infty} 2 \gamma v A_{p}\left(t^{\prime}\right) A_{q}\left(t^{\prime}\right) d t^{\prime},
$$

and the conversion efficiency is

$$
\eta^{(1)} \equiv \frac{\int_{-\infty}^{\infty}\left|A_{i}^{(1)}\left(-\frac{L}{2}, t^{\prime}\right)\right|^{2} d t^{\prime}}{\int_{-\infty}^{\infty}\left|A_{s}^{(1)}\left(-\frac{L}{2}, t^{\prime}\right)\right|^{2} d t^{\prime}}=\left[\int_{-\infty}^{\infty}\left|2 \gamma v A_{p}\left(t^{\prime}\right) A_{q}\left(t^{\prime}\right)\right| d t^{\prime}\right]^{2},
$$

assuming that either of the pump pulses $A_{p}$ and $A_{q}$ is much shorter than the input signal $F$ and the medium is long enough to contain the signal. Conceptually, the short pump pulses take a "snapshot" of the signal spatial profile, which is reproduced as the idler. Since the idler has the same spatial profile as the signal but propagates backwards, the time profile is reversed.

To summarize, in order to perform accurate SPC, the following conditions should be satisfied:

$$
\frac{L}{v}>>T_{s}>>\left(T_{p} \text { or } T_{q}\right)>>\frac{d}{v_{x}},
$$

where $T_{s}$ is the signal pulse width, and $T_{p}$ and $T_{q}$ are the pulse widths of the two pumps. 


\section{High conversion efficiency with signal amplification}

When the conversion efficiency is high, mixing of the pump and the generated idler can also amplify the signal, as in the case of parametric amplification. In this section we derive accurate expressions for the output idler and the conversion efficiency in such a case. Assuming that the pump pulses are short, unchirped and undepleted, and phase modulation terms are neglected, we can derive a closed-form solution for the conversion efficiency. Eqs. (3) and (4) then become

$$
\begin{aligned}
v \frac{\partial A_{s}(z, t)}{\partial z}+\frac{\partial A_{s}(z, t)}{\partial t} & =j g(t) A_{i}^{*}(z, t), \\
-v \frac{\partial A_{i}(z, t)}{\partial z}+\frac{\partial A_{i}(z, t)}{\partial t} & =j g(t) A_{s}^{*}(z, t), \\
\text { where } g(t) & =2 \gamma v A_{p}(t) A_{q}(t) .
\end{aligned}
$$

We first take the complex conjugate of Eq. (14),

$$
-v \frac{\partial A_{i}^{*}(z, t)}{\partial z}+\frac{\partial A_{i}^{*}(z, t)}{\partial t}=-j g^{*}(t) A_{s}(z, t),
$$

and let $\tilde{A}_{s}$ and $\tilde{A}_{i}$ be the Fourier transforms of $A_{s}$ and $A_{i}^{*}$ with respect to $z$ respectively,

$$
\begin{aligned}
& \tilde{A}_{s}(\kappa, t)=\int_{-\infty}^{\infty} A_{s}(z, t) \exp (-j \kappa z) d z, \\
& \tilde{A}_{i}(\kappa, t)=\int_{-\infty}^{\infty} A_{i}^{*}(z, t) \exp (-j \kappa z) d z .
\end{aligned}
$$

Note that $\tilde{A}_{i}$ is the Fourier transform of the complex conjugate of $A_{i}$. Eqs. (13) and (16) become

$$
\begin{aligned}
j \kappa v \tilde{A}_{s}+\frac{\partial \tilde{A}_{s}}{\partial t} & =j g(t) \tilde{A}_{i}, \\
-j \kappa v \tilde{A}_{i}+\frac{\partial \tilde{A}_{i}}{\partial t} & =-j g^{*}(t) \tilde{A}_{s} .
\end{aligned}
$$

We multiply both sides of Eq. (19) by $\exp (j \kappa v t)$ and both sides of Eq. (20) by $\exp (-j \kappa v t)$,

$$
\begin{aligned}
\exp (j \kappa v t)\left(j \kappa v \tilde{A}_{s}+\frac{\partial \tilde{A}_{s}}{\partial t}\right) & =j g(t) \exp (j \kappa v t) \tilde{A}_{i}, \\
\exp (-j \kappa v t)\left(-j \kappa v \tilde{A}_{i}+\frac{\partial \tilde{A}_{i}}{\partial t}\right) & =-j g^{*}(t) \exp (-j \kappa v t) \tilde{A}_{s},
\end{aligned}
$$

or equivalently,

$$
\begin{aligned}
\frac{\partial}{\partial t}\left[\exp (j \kappa v t) \tilde{A}_{s}\right] & =j g(t) \exp (j \kappa v t) \tilde{A}_{i}, \\
\frac{\partial}{\partial t}\left[\exp (-j \kappa v t) \tilde{A}_{i}\right] & =-j g^{*}(t) \exp (-j \kappa v t) \tilde{A}_{s} .
\end{aligned}
$$

Then we make another set of substitutions,

$$
\begin{aligned}
& A(\kappa, t)=\exp (j \kappa v t) \tilde{A}_{s}=\exp (j \kappa v t) \int_{-\infty}^{\infty} A_{s}(z, t) \exp (-j \kappa z) d z \\
& B(\kappa, t)=\exp (-j \kappa v t) \tilde{A}_{i}=\exp (-j \kappa v t) \int_{-\infty}^{\infty} A_{i}^{*}(z, t) \exp (-j \kappa z) d z,
\end{aligned}
$$


Eqs. (23) and (24) become

$$
\begin{aligned}
& \frac{\partial A}{\partial t}=j g(t) \exp (2 j \kappa v t) B \\
& \frac{\partial B}{\partial t}=-j g^{*}(t) \exp (-2 j \kappa v t) A .
\end{aligned}
$$

The exponential terms on the right-hand side have a frequency $2 \kappa v$. To estimate the magnitude of this frequency, it is best to first consider the linear propagation of the signal and idler envelopes, before wave mixing occurs,

$$
\begin{aligned}
v \frac{\partial A_{s}}{\partial z}+\frac{\partial A_{s}}{\partial t} & =0 \\
-v \frac{\partial A_{i}}{\partial z}+\frac{\partial A_{i}}{\partial t} & =0 .
\end{aligned}
$$

Fourier transforms in $z$ as well as $t$ give the dispersion relation for the envelopes,

$$
|\kappa v|=|\Omega|,
$$

which is consistent with the definition of group velocity, $v=\frac{d \omega}{d k} . \Omega$ is the frequency variable in taking the temporal Fourier transform of the signal and idler envelopes, and has a maximum magnitude $\sim 1 / T_{s}$. From Eqs. (27) and (28) it can be observed that wave mixing does not alter the spatial bandwidth of the envelopes, therefore $\kappa$ has the same order of magnitude throughout, and $\kappa v \sim 1 / T_{s}<<\left(1 / T_{p}\right.$ or $\left.1 / T_{q}\right) . g(t)$ has a duration shorter than both $T_{p}$ and $T_{q}$, so $\exp (2 j \kappa v t)$ oscillates relatively slowly compared to $g(t)$. Say $g(t)$ is centered at $t=0$, we can then make the assumption,

$$
g(t) \exp (2 j \kappa v t) \approx g(t)
$$

The coupled-mode equations (27) and (28) become

$$
\begin{aligned}
& \frac{\partial A}{\partial t}=j g(t) B \\
& \frac{\partial B}{\partial t}=-j g^{*}(t) A .
\end{aligned}
$$

The initial condition is

$$
\begin{aligned}
& A_{s}\left(z,-\frac{L}{2 v}\right)=F\left(-\frac{L}{2 v}-\frac{z}{v}\right), \\
& A_{i}\left(z,-\frac{L}{2 v}\right)=0 .
\end{aligned}
$$

The initial condition for $A$ and $B$ can then be obtained from the substitutions, Eqs. (25) and (26). Define $g(t)=|g(t)| \exp j \theta(t)$, and assume that $\theta(t)$ is a constant. Eqs. (33) and (34) can now be solved to give

$$
\begin{aligned}
& A(\kappa, t)=A\left(\kappa,-\frac{L}{2 v}\right) \cosh \left[\int_{-\frac{L}{2 v}}^{t}\left|g\left(t^{\prime}\right)\right| d t^{\prime}\right] \\
& B(\kappa, t)=-j A\left(\kappa,-\frac{L}{2 v}\right) \exp (-j \theta) \sinh \left[\int_{-\frac{L}{2 v}}^{t}\left|g\left(t^{\prime}\right)\right| d t^{\prime}\right] .
\end{aligned}
$$


The final solution for $A_{s}$ and $A_{i}$ is

$$
\begin{aligned}
& A_{s}(z, t)=F\left(t-\frac{z}{v}\right) \cosh \left[\int_{-\frac{L}{2 v}}^{t}\left|g\left(t^{\prime}\right)\right| d t^{\prime}\right], \\
& A_{i}(z, t)=j F^{*}\left(-t-\frac{z}{v}\right) \exp (j \theta) \sinh \left[\int_{-\frac{L}{2 v}}^{t}\left|g\left(t^{\prime}\right)\right| d t^{\prime}\right] .
\end{aligned}
$$

As the idler exits the medium at $z=-\frac{L}{2}$ and $t=\frac{L}{2 v}$, the pump pulses have long gone, hence the upper integral limit can be effectively replaced by $\infty$. The lower limit can also be replaced by $-\infty$, since the pump pulses have not arrived when the signal enters the medium at $t=-\frac{L}{2 v}$. Hence

$$
A_{i}\left(-\frac{L}{2}, t\right)=j F^{*}\left(-t+\frac{L}{2 v}\right) \exp (j \theta) \sinh \left[\int_{-\infty}^{\infty}\left|g\left(t^{\prime}\right)\right| d t^{\prime}\right] .
$$

This solution is consistent with Eq. (10), the first-order approximation in the limit of small gain. The conversion efficiency is

$$
\eta \equiv \frac{\int_{-\infty}^{\infty}\left|A_{i}\left(-\frac{L}{2}, t^{\prime}\right)\right|^{2} d t^{\prime}}{\int_{-\infty}^{\infty}\left|A_{s}\left(-\frac{L}{2}, t^{\prime}\right)\right|^{2} d t^{\prime}}=\sinh ^{2}\left[\int_{-\infty}^{\infty}\left|2 \gamma \nu A_{p}\left(t^{\prime}\right) A_{q}\left(t^{\prime}\right)\right| d t^{\prime}\right] .
$$

This result shows the exponential dependence of the conversion efficiency on the cross fluence of the two pump pulses.

\section{Cross-phase modulation compensation}

With the undepleted pump approximation, the main nonlinear effect besides wave mixing is the cross-phase modulation on the signal and the idler imposed by the strong pump. Mathematically this can be observed from Eq. (3) and Eq. (4), where the XPM terms are the largest apart from the wave mixing terms. These effects are previously neglected in deriving Eq. (42).

With XPM terms included, the coupled-mode equations become

$$
\begin{aligned}
v \frac{\partial A_{s}(z, t)}{\partial z}+\frac{\partial A_{s}(z, t)}{\partial t} & =j g(t) A_{i}^{*}(z, t)+j c(t) A_{s}(z, t), \\
-v \frac{\partial A_{i}(z, t)}{\partial z}+\frac{\partial A_{i}(z, t)}{\partial t} & =j g(t) A_{s}^{*}(z, t)+j c(t) A_{i}(z, t), \\
\text { where } g(t) & =2 \gamma v A_{p}(t) A_{q}(t), \\
c(t) & =2 \gamma v\left[\left|A_{p}(t)\right|^{2}+\left|A_{q}(t)\right|^{2}\right] .
\end{aligned}
$$

XPM effects are detrimental to the SPC efficiency and accuracy if a high conversion efficiency is desired, as it introduces a time-dependent detuning factor to the wave mixing process.

To solve Eqs. (43) and (44), we follow similar procedures as in the previous section by performing Fourier transform with respect to $z$ and making the following substitutions,

$$
\begin{aligned}
A(\kappa, t) & =\exp \left[j \kappa v t-j \int_{-\infty}^{t} c\left(t^{\prime}\right) d t^{\prime}\right] \int_{-\infty}^{\infty} A_{s}(z, t) \exp (-j \kappa z) d z \\
B(\kappa, t) & =\exp \left[-j \kappa v t+j \int_{-\infty}^{t} c\left(t^{\prime}\right) d t^{\prime}\right] \int_{-\infty}^{\infty} A_{i}^{*}(z, t) \exp (-j \kappa z) d z .
\end{aligned}
$$

We obtain the following,

$$
\begin{aligned}
& \frac{\partial A}{\partial t}=j g(t) \exp \left[-2 j \int_{-\infty}^{t} c\left(t^{\prime}\right) d t^{\prime}\right] B \\
& \frac{\partial B}{\partial t}=-j g^{*}(t) \exp \left[2 j \int_{-\infty}^{t} c\left(t^{\prime}\right) d t^{\prime}\right] A .
\end{aligned}
$$


Eqs. (49) and (50) are difficult to solve analytically, but a special case exists when the phase of $g(t)$ exactly cancels the XPM term,

$$
\theta(t)=\theta_{0}+2 \int_{-\infty}^{t} c\left(t^{\prime}\right) d t^{\prime} .
$$

Eqs. (49) and (50) are then reduced to

$$
\begin{aligned}
& \frac{\partial A}{\partial t}=j|g(t)| \exp \left(j \theta_{0}\right) B \\
& \frac{\partial B}{\partial t}=-j|g(t)| \exp \left(-j \theta_{0}\right) A
\end{aligned}
$$

The general solution is

$$
\begin{aligned}
& A_{s}(z, t)=F\left(t-\frac{z}{v}\right) \exp \left[j \int_{-\infty}^{t} c\left(t^{\prime}\right) d t^{\prime}\right] \cosh \left[\int_{-\infty}^{t}\left|g\left(t^{\prime}\right)\right| d t^{\prime}\right], \\
& A_{i}(z, t)=j F^{*}\left(-t-\frac{z}{v}\right) \exp \left[j \theta_{0}+j \int_{-\infty}^{t} c\left(t^{\prime}\right) d t^{\prime}\right] \sinh \left[\int_{-\infty}^{t}\left|g\left(t^{\prime}\right)\right| d t^{\prime}\right],
\end{aligned}
$$

and the output idler is

$$
A_{i}\left(-\frac{L}{2}, t\right)=j F^{*}\left(-t+\frac{L}{2 v}\right) \exp \left[j \theta_{0}+j \int_{-\infty}^{\infty} c\left(t^{\prime}\right) d t^{\prime}\right] \sinh \left[\int_{-\infty}^{\infty}\left|g\left(t^{\prime}\right)\right| d t^{\prime}\right] .
$$

This solution is the same as Eq. (41), the output idler without considering XPM, apart from a constant phase term $\exp \left[\int_{-\infty}^{\infty} c\left(t^{\prime}\right) d t^{\prime}\right]$, which does not affect the pulse waveform. If we let $A_{p}(t)=\left|A_{p}(t)\right| \exp \left[j \theta_{p}(t)\right]$ and $A_{q}(t)=\left|A_{q}(t)\right| \exp \left[j \theta_{q}(t)\right]$, then from Eq. (51) the actual phase adjustments to the pump pulses are given by

$$
\theta_{p}(t)+\theta_{q}(t)=\theta_{0}+4 \gamma v \int_{-\infty}^{t}\left|A_{p}\left(t^{\prime}\right)\right|^{2}+\left|A_{q}\left(t^{\prime}\right)\right|^{2} d t^{\prime} .
$$

Qualitatively, by adjusting the phases of the pump pulses according to Eq. (57), we can utilize the wave mixing process to introduce phase variations to the signal and the idler, so that the cross-phase modulation can be exactly canceled. In practice, the phase variation of the pump pulses can be introduced by various pulse shaping methods, for example, using a $4 \mathrm{f}$ pulse shaper [12]. The phase correction can be introduced to either or both of the pump pulses as long as the condition in Eq. (57) is satisfied.

\section{Numerical analysis}

To verify our derivations, we obtain numerical solutions of Eqs. (43) and (44) by a multi-scale approach. In this approach successively higher-order solutions are obtained by substituting lower-order solutions into the right-hand side of the equations, until convergence is reached. For the following simulations, the pump and the input signal are assumed to be

$$
\begin{aligned}
A_{p}(t)=A_{q}(t) & =\exp \left(-\frac{t^{2}}{2 T_{p}^{2}}\right), \\
F(\tau) & =A_{s 0}\left\{\exp \left[-\frac{1+j}{2}\left(\frac{\tau+2 T_{s}}{T_{s}}\right)^{2}\right]+\frac{1}{2} \exp \left[-\frac{1}{2}\left(\frac{\tau-2 T_{s}}{T_{s}}\right)^{2}\right]\right\} .
\end{aligned}
$$

To confirm that the SPC process is still accurate when the conversion efficiency is high, we first consider the case in which XPM is neglected. Figure 2 shows a plot of the amplitude 
and the phase of the output idler pulse envelope $A_{i}\left(-\frac{L}{2}, t\right)$ compared with the input signal $A_{s}\left(-\frac{L}{2}, t\right)$, using parameters similar to Ref. [9] and polydiacetylene, a material with the highest off-resonant third-order nonlinearity reported [11], as the wave mixing medium. The conversion efficiency is $100 \%$ with a total pump energy of only $12.8 \mathrm{~nJ}$ from the numerical analysis. From Fig. 2 it is clear that the output idler is an exact, time-reversed and phase-conjugated replica of the input signal.
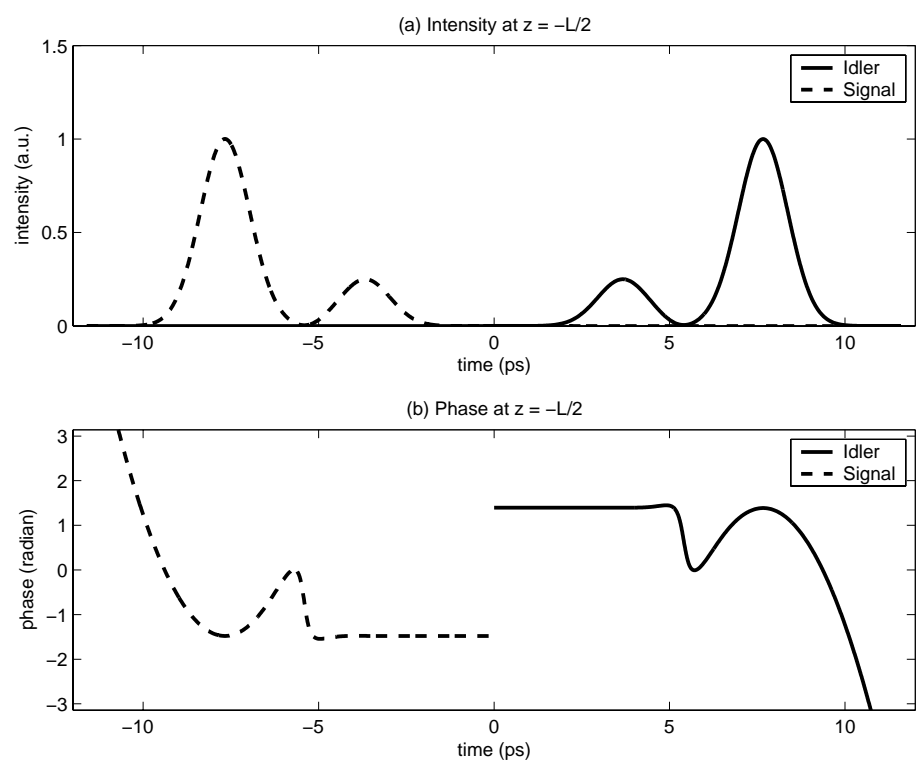

Fig. 2. (a) Amplitude and (b) phase of output idler (solid lines) $A_{i}\left(-\frac{L}{2}, t\right)$ compared with input signal (dash lines) $A_{s}\left(-\frac{L}{2}, t\right)$. XPM is neglected in this example. As predicted, the output idler is time-reversed and phase-conjugated with respect to the input signal. Parameters used are $n_{2}=1 \times 10^{-11} \mathrm{~cm}^{2} / \mathrm{W}, n_{0}=1.7, \lambda_{0}=800 \mathrm{~nm}, L=2 \mathrm{~mm}, d=5 \mu \mathrm{m}, T_{s}=$ $1 \mathrm{ps}, T_{p}=100 \mathrm{fs}, E_{p}=12.8 \mathrm{~nJ}$, pump fluence $=\frac{E_{p}}{L d}$. Conversion efficiency is $100 \%$.

\subsection{Conversion efficiency}

Figure 3 is a plot of conversion efficiencies against total pump energy obtained from theory and simulations, using the same parameters as for the previous numerical example. The dotted curve is a plot of Eq. (11), the result from Ref. [1]. The solid curve is a plot of Eq. (42), the conversion efficiency obtained by including signal amplification but neglecting XPM. The crosses are results from a numerical simulation of Eqs. (13) and (14), validating the closed-form solution we derive. The triangles are results from a numerical simulation of Eqs. (3) and (4), which also include phase modulation terms. It clearly shows that XPM becomes detrimental to the conversion efficiency as the pump energy increases. Finally, the circles are a numerical simulation that includes all nonlinear terms and XPM compensation according to Eq. (57). The numerical results confirm the accuracy of our conversion efficiency derivation, demonstrates the detrimental XPM effect on conversion efficiency, and proves that our compensation method can indeed undo the XPM effect. 


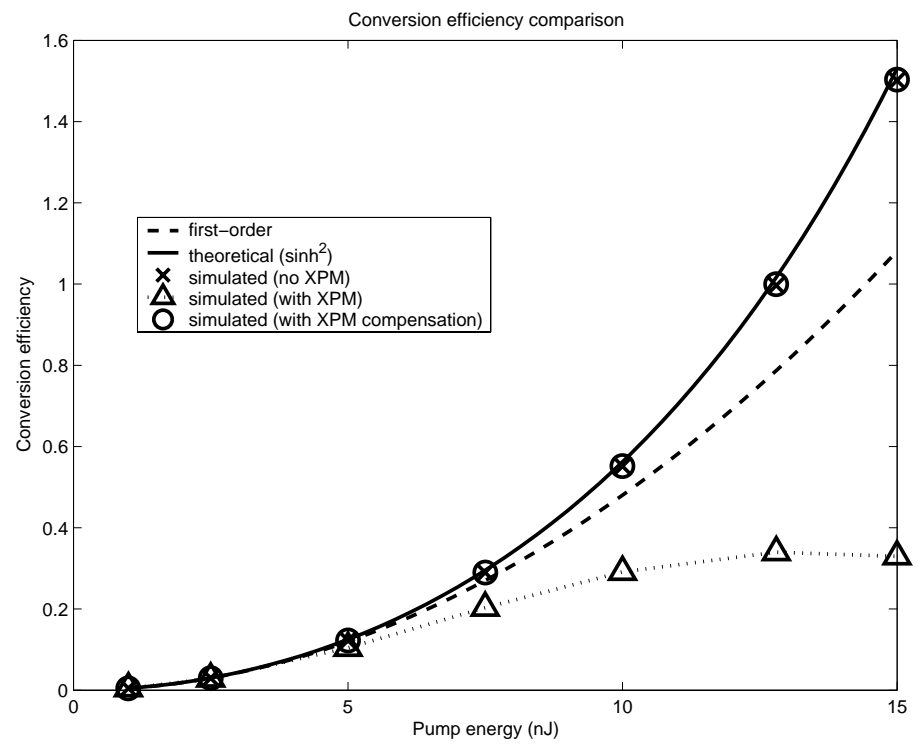

Fig. 3. Conversion efficiencies from simulations compared with predictions from first-order analysis and coupled-mode theory. Simulation results agree well with coupled-mode theory. See caption of Fig. 2 for parameters used.

\subsection{Demonstration of cross-phase modulation compensation}

Figure 4(a) and (b) plot the output idler $A_{i}\left(-\frac{L}{2}, t\right)$ compared with the SPC of the input signal $A_{s}^{*}\left(-\frac{L}{2},-t\right)$, with XPM included, using the same parameters as before. The efficiency is reduced from $100 \%$ to $34 \%$ and the accuracy of the SPC operation suffers due to the XPM effect. Figure 4(c) and (d) plots the same data, but with the phase of the pump pulses adjusted according to Eq. (57) and plotted in Fig. 5. The accuracy of the SPC operation is restored by the XPM compensation, and the efficiency is back to $100 \%$.

\section{Beyond the basic assumptions}

\subsection{Pump depletion}

All of our derivations so far assume that the pump is undepleted. If the signal becomes comparable to the pump, then the pump can no longer sustain a fixed gain, which begins to depend on the signal field across $z$. Mathematically this means that the right-hand sides of Eqs. (1) and (2) become comparable to the left-hand sides. In this case the pump would be depleted, and we can no longer expect the SPC operation to be accurate. To avoid pump depletion we therefore require the right-hand sides of Eqs. (1) and (2) to be much smaller than the left-hand sides, or roughly speaking,

$$
\begin{aligned}
\left|A_{p}\right| & >2 \gamma\left|A_{s}\right|\left|A_{i}\right|\left|A_{q}\right| d \\
E_{s} & <<\frac{n_{0} d T_{s}}{\eta_{0} \gamma \sqrt{\eta}} .
\end{aligned}
$$

where $E_{s}$ is the signal energy and $\eta_{0}$ is the free-space impedance. The signal energies should be much smaller than the rough signal energy upper limits established by Eq. (61) in order to avoid pump depletion. A low signal energy also avoids distortion due to SPM. 

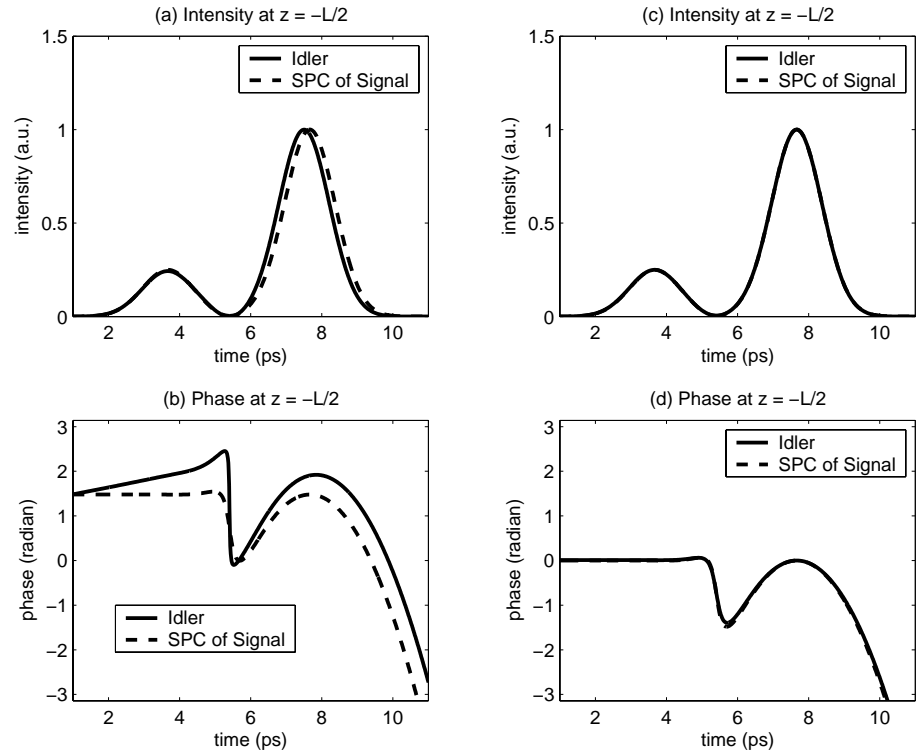

Fig. 4. (a) and (b) plot the normalized amplitude and phase of the output idler $A_{i}\left(-\frac{L}{2}, t\right)$ compared to the SPC of the input signal $A_{s}^{*}\left(-\frac{L}{2},-t\right)$ respectively when XPM is present. The amplitude plots are normalized with respect to their peaks. The output idler is distorted and the conversion efficiency is only $34 \%$, much lower than the theoretical efficiency $100 \%$. (c) and (d) plot the same data, but with XPM compensation. The efficiency is back to $100 \%$ and the accuracy is restored.
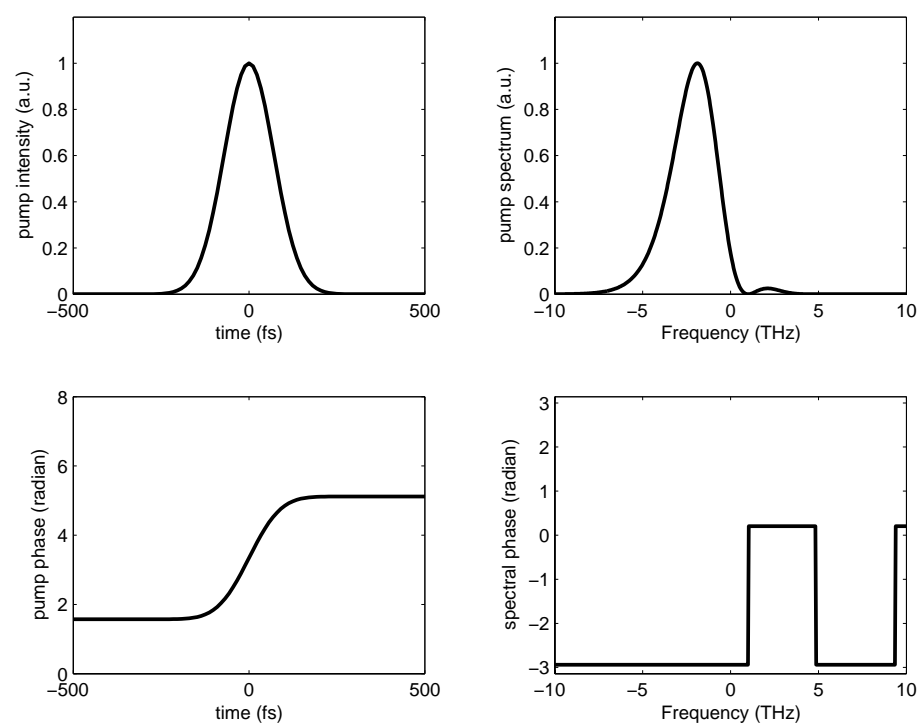

Fig. 5. Plots of amplitude and phase of one pump pulse with ideal phase adjustment according to Eq. (57) in time and frequency domain. Top-left: temporal envelope; Bottom-left: temporal phase; Top-right: envelope spectrum; Bottom-right: spectral phase. The simple pulse shape should be easily produced by many pulse shaping methods. 
To investigate the effect of pump depletion, we perform three-dimensional simulations in $x$, $z, t$ by numerically solving Eqs. (1), (2), (3) and (4) simultaneously.

The first example assumes the same parameters as before, with a signal energy of $1 \mathrm{pJ}$, much below the pump depletion limit, calculated to be $1 \mathrm{~nJ}$ from Eq. (61). XPM is included along with XPM compensation. Figure 6 is a movie that shows the evolution of the wave mixing process. The conversion efficiency from the simulation drops slightly to $92 \%$ due to a finite medium thickness. However, the SPC process still remains accurate with the inclusion of the $x$ dimension.

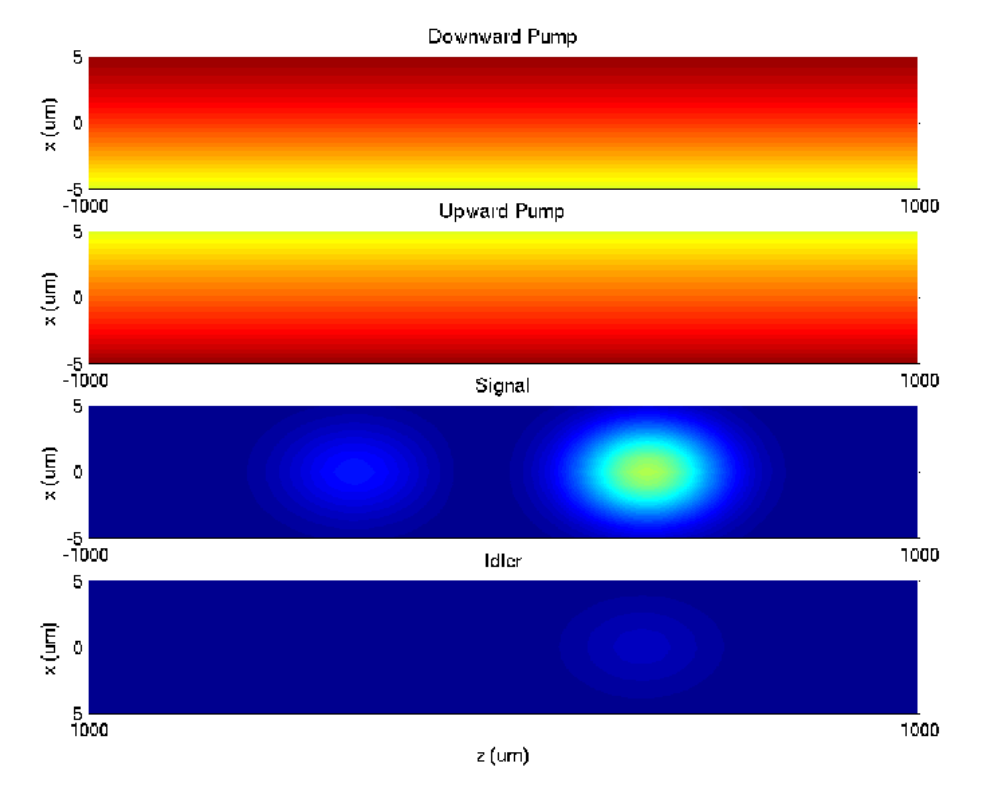

Fig. 6. A movie that shows the evolution of the two pumps, signal and idler, with a signal energy below the pump depletion limit. See caption of Fig. 2 for parameters used. The signal energy is $1 \mathrm{pJ}$.

Figure 7 is another movie of the same example but with a signal energy of $5 \mathrm{~nJ}$, much above the pump depletion limit $1 \mathrm{~nJ}$, and Fig. 8 plots the output idler from the same simulation. As can be seen from the movie, the pump pulses are highly depleted, and from Fig. 8 it can be seen that the top of the idler is flattened due to gain saturation. The conversion efficiency is reduced to $32 \%$.

\subsection{Other non-ideal conditions}

Besides pump depletion, other non-ideal conditions also affect the accuracy of the SPC process. If the pump pulses are not short enough, then from the first-order solution in Ref. [1] it can be seen that the output pulse becomes the convolution of the pump and the signal. The medium also needs to be long enough to contain the whole signal pulse, otherwise the output idler will be truncated.

If the medium is thick, the $x$ dimension can no longer be ignored. $A_{p}(t)$ and $A_{q}(t)$ should be replaced by $A_{p}\left(t+x / v_{x}\right)$ and $A_{q}\left(t-x / v_{x}\right)$ respectively, which do not directly affect the generated idler temporal pulse shape. However, the idler beam will acquire additional transverse patterns in $x$, which will distort the pulse shape if diffraction or waveguiding is also taken into account. Another problem with a thick medium is that SPM will chirp the pump pulses and reduce the SPC efficiency and accuracy. That said, since SPM is not directly involved with the 


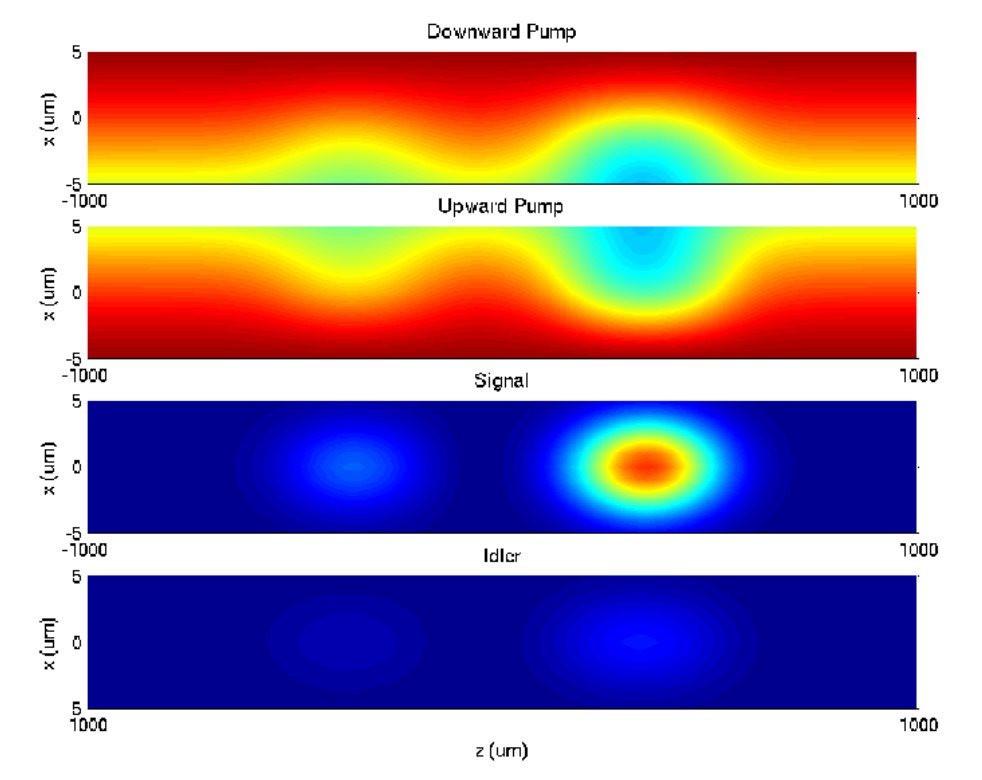

Fig. 7. Similar to Fig. 6, but with a signal energy of 5 nJ, much above the pump depletion limit.
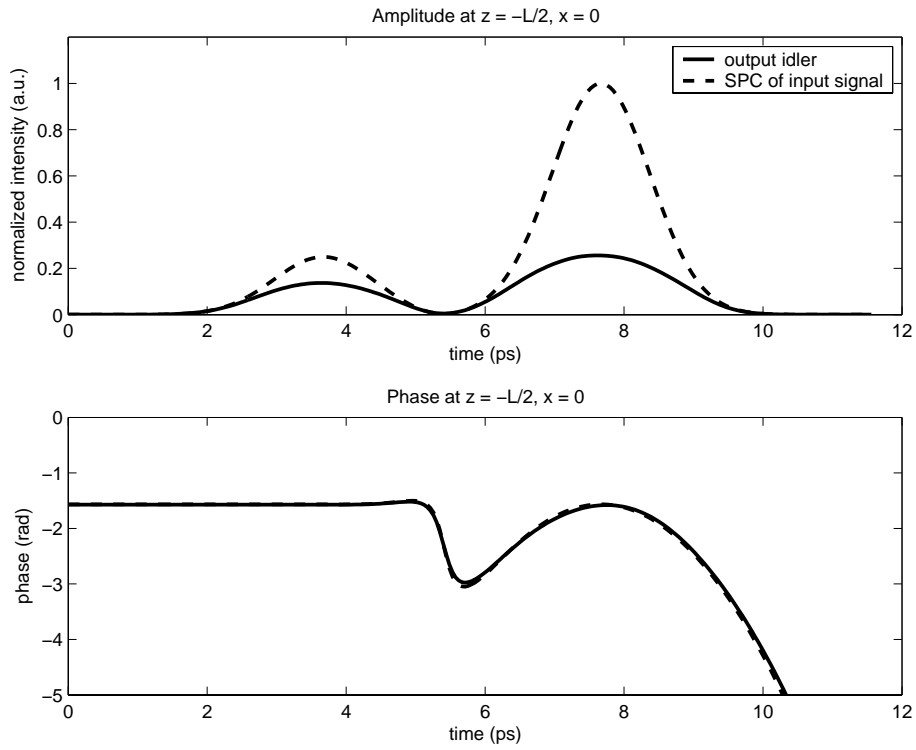

Fig. 8. Amplitude and phase of output idler and input signal for the wave mixing process shown in Fig. 7 to demonstrate the effect of pump depletion. 
wave mixing process, it can be precompensated by pulse shaping if it becomes a problem.

\section{Summary}

In summary, we have derived new solutions for the spectral phase conjugation process by fourwave mixing. When signal amplification is significant, the SPC process has been shown to remain accurate and achieve higher efficiency. We have also proposed a method to compensate for XPM, which severely reduces the efficiency and accuracy of SPC, by adjusting the phases of the pump pulses accordingly. Numerical analysis confirms our theoretical predictions, and experimental verification will be the focus of our future research. With a higher predicted conversion efficiency and an effective method to compensate for XPM without sacrificing accuracy, we are hopeful that SPC by four-wave mixing can finally be experimentally implemented and utilized for real-world applications.

\section{Acknowledgments}

This work was supported primarily by the Engineering Research Centers Program of the National Science Foundation under Award Number EEC-9402726. 